\title{
Prevalence and Predictors of Prediabetes And Diabetes Mellitus In Kenya: Results from a National Survey
}

Martin Mwangi1,2, Joseph Kibachio², Shukri Mohamed ${ }^{3}$, Martin K Mutua ${ }^{3}$, Abubakar Hussein ${ }^{1,2}$ Zachary Ndegwa², Scholastica Mwende², Catherine Kyobutungi $i^{3}$

1. Field Epidemiology and Laboratory Training Programme, Ministry of Health, Kenya

2. Non-communicable Disease Division, Ministry of Health, Kenya

3. African Population and Health Research Center, Nairobi, Kenya

\section{Introduction}

High blood glucose is the 3rd highest risk factor for premature mortality

Prediabetes is the precursor stage to diabetes mellitus

In Kenya the number of undiagnosed diabetics is estimated at 287.7 per 1000 population (IDF, 2013)

Screening of diabetes is not routine in clinical practice

$60 \%$ of cases missed at presentation

This study aimed to determine the prevalence of prediabetes, diabetes and identify the predictors of prediabetes in adults in Kenya

的

Methods \& Materials Study Site

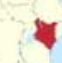

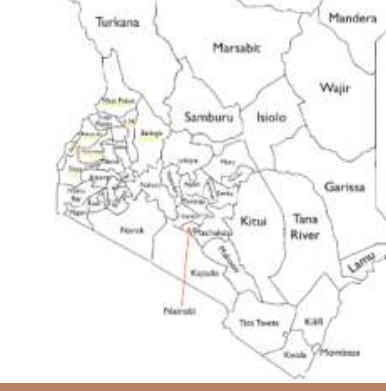

- A population-based, nationwide cross-sectional survey was conducted between April and June 2015 - Using the WHO Stepwise approach to chronic disease risk factor surveillance - Participants aged 18-69 years Definitions

- Prediabetes - impaired fasting blood glucose of $6.1 \mathrm{mmol} / /$ to less than $7 \mathrm{mmol} / \mathrm{l}$

- Diabetes is impaired fasting blood glucose of $7 \mathrm{mmol} / \mathrm{l}$ or more Analysis

- Analysis of STEPS dataset was done

- Descriptive statistics were used to determine the prevalence of prediabetes and diabetes

- Logistic regression was used to identify predictors of

prediabetes and diabetes

\section{Results}

The study had 4500 respondents but 4163 respondents were included in the analysis Females (51\%) age group 18-29 (46\%) and lived in rural areas (62\%)

- About $42 \%$ had secondary education and above, $39 \%$ were self-employed and $66 \%$ were married

Socio demographics characteristics of the participants

\begin{tabular}{|c|c|c|c|c|}
\hline Variable & $\begin{array}{l}\text { Normal } \mathrm{n} \\
(\%)\end{array}$ & $\begin{array}{l}\text { Prediabetes } \\
\mathrm{n}(\%)\end{array}$ & $\begin{array}{l}\text { Diabetes n } \\
\text { (\%) }\end{array}$ & $\begin{array}{c}\text { Total } \\
\mathbf{n}\end{array}$ \\
\hline \multicolumn{5}{|l|}{ Sex } \\
\hline Male & $1,877(93)$ & $119(6)$ & $26(1)$ & 2,022 \\
\hline Female & $1,922(90)$ & $168(8)$ & $51(2)$ & 2,141 \\
\hline \multicolumn{5}{|l|}{ Age groups } \\
\hline $18-29$ & $1,833(95)$ & $90(5)$ & $9(0)$ & 1,932 \\
\hline $30-39$ & 878 (91) & 71 (7) & 13(1) & 962 \\
\hline $40-49$ & 581 (91) & 49 (8) & 19 (3) & 648 \\
\hline 50-59 & $325(80)$ & 50 (12) & 29 (7) & 404 \\
\hline 60-69 & $182(84)$ & 27 (12) & $7(3)$ & 217 \\
\hline \multicolumn{5}{|l|}{ Education level } \\
\hline $\begin{array}{l}\text { No formal } \\
\text { education }\end{array}$ & $441(86)$ & $54(11)$ & $16(3)$ & 510 \\
\hline $\begin{array}{c}\text { Primary } \\
\text { incomplete }\end{array}$ & 909 (93) & 55 (6) & $16(2)$ & 981 \\
\hline $\begin{array}{c}\text { Primary } \\
\text { complete }\end{array}$ & 850 (93) & 49 (5) & $18(2)$ & 917 \\
\hline $\begin{array}{l}\text { Secondary and } \\
\text { above }\end{array}$ & 1599 (91) & $129(7)$ & $27(2)$ & 1755 \\
\hline \multicolumn{5}{|l|}{ Residence } \\
\hline Rural & $2,377(92)$ & 179 (7) & $40(2)$ & 2,597 \\
\hline Urban & 1,422 (91) & 108 (7) & 37 (2) & 1,566 \\
\hline \multicolumn{5}{|l|}{ Marital status } \\
\hline Not married & $923(96)$ & $36(4)$ & $5(1)$ & 964 \\
\hline Married & $2480(90)$ & 206 (8) & 59 (2) & 2745 \\
\hline $\begin{array}{c}\text { Formerly } \\
\text { married }\end{array}$ & $396(87)$ & $46(10)$ & $13(3)$ & 454 \\
\hline
\end{tabular}

The prevalence for prediabetes was $7 \%$ (5.9, 8.0)

The prevalence of diabetes was $2 \%(1.4,2.5)$ - Prevalence of prediabetes was higher in women $(7.8 \%)$, those aged 50-59 (12\%), those with no formal education (11\%)

Prevalence of prediabetes and diabetes

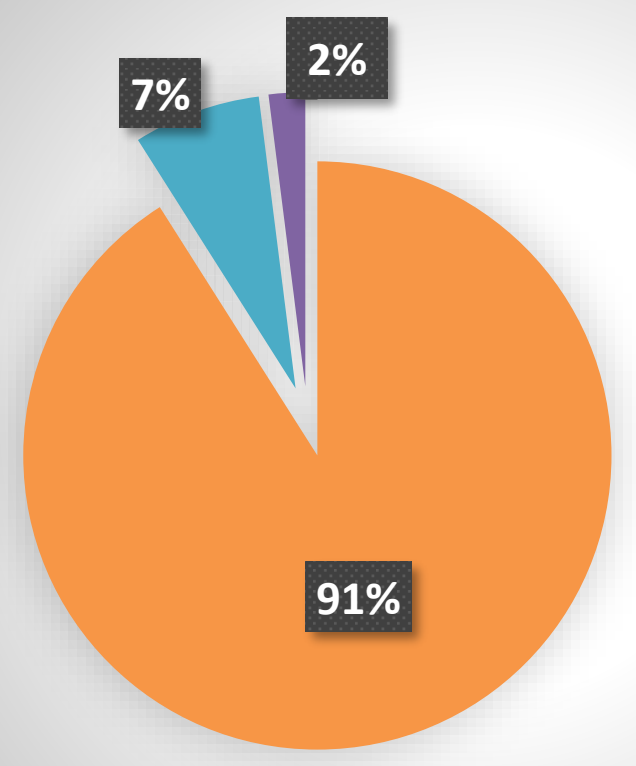

normal - Prediabetes n Diabetes

Bivariate and multivariate analysis of predictors for pre-diabetes among adults in Kenya 2015

\begin{tabular}{|c|c|c|c|c|}
\hline & \multicolumn{2}{|c|}{ Bivariate } & \multicolumn{2}{|c|}{ Multivariate } \\
\hline Pre-diabetics & $\begin{array}{l}\text { Odds Ratio } \\
(95 \% \text { Cl) }\end{array}$ & $P>z$ & $\begin{array}{l}\text { Odds Ratio } \\
(95 \% \text { Cl) }\end{array}$ & $P>z$ \\
\hline \multicolumn{5}{|l|}{ Age group } \\
\hline $18-29$ & 1.00 & & 1.00 & \\
\hline $30-39$ & $\begin{array}{c}1.63 \\
(1.18,2.24)\end{array}$ & 0.003 & $\begin{array}{c}1.42 \\
(1.02,1.98)\end{array}$ & 0.040 \\
\hline $40-49$ & $\begin{array}{c}1.66 \\
(1.16,2.38)\end{array}$ & 0.006 & $\begin{array}{c}1.38 \\
(0.94,2.02)\end{array}$ & 0.098 \\
\hline 50-59 & $\begin{array}{c}2.92 \\
(2.03,4.19)\end{array}$ & $<0.001$ & $\begin{array}{c}2.22 \\
(1.49,3.32)\end{array}$ & $<0.001$ \\
\hline 60-69 & $\begin{array}{c}2.91 \\
(1.85,4.59)\end{array}$ & $<0.001$ & $\begin{array}{c}2.41 \\
(1.47,3.95)\end{array}$ & 0.001 \\
\hline \multicolumn{5}{|c|}{ Episodic drinking } \\
\hline $\begin{array}{l}\text { Non-heavy } \\
\text { drinking }\end{array}$ & $\begin{array}{c}2.02 \\
(1.27,3.2)\end{array}$ & 0.003 & $\begin{array}{c}2.11 \\
(1.29,3.45)\end{array}$ & 0.003 \\
\hline \multicolumn{5}{|l|}{ BMI } \\
\hline Normal & 1.00 & & 1.00 & \\
\hline Overweight & $\begin{array}{c}1.42 \\
(1.04,1.94)\end{array}$ & 0.027 & $\begin{array}{c}1.42 \\
(1.01,2.00)\end{array}$ & 0.043 \\
\hline Obese & $\begin{array}{c}2.68 \\
(1.91,3.76)\end{array}$ & $<0.001$ & $\begin{array}{c}2.46 \\
(1.66,3.64)\end{array}$ & $<0.001$ \\
\hline \multicolumn{5}{|l|}{ Waist Hip Ratio } \\
\hline Normal & 1.00 & & 1.00 & \\
\hline $\begin{array}{l}\text { Centrally } \\
\text { obese }\end{array}$ & $\begin{array}{c}1.94 \\
(1.51,2.48)\end{array}$ & $<0.001$ & $\begin{array}{c}1.44 \\
(1.09,1.92)\end{array}$ & 0.011 \\
\hline
\end{tabular}

The significant age-diabetes association was from age group 30-39 at 2.87 times at risk with an increased risk as the age increases The females were at risk with 1.87 times to be associated to diabetes

Physical inactivity had odds of $\mathbf{2 . 7 8}$ more likely to be associated with diabetes

Bivariate and multivariate analysis of predictors for diabetes among adults in Kenya 2015

\begin{tabular}{|c|c|c|c|c|}
\hline & \multicolumn{2}{|c|}{ Bivariate } & \multicolumn{2}{|c|}{ Multivariate } \\
\hline Diabetics & $\begin{array}{l}\text { Odds Ratio } \\
(95 \% \text { Cl) }\end{array}$ & $P>z$ & $\begin{array}{l}\text { Odds Ratio } \\
(95 \% \text { Cl) }\end{array}$ & $P>Z$ \\
\hline \multicolumn{5}{|l|}{ Age group } \\
\hline $18-29$ & 1.00 & & 1.00 & \\
\hline 30-39 & $\begin{array}{c}2.77(1.19 \\
6.45)\end{array}$ & $\begin{array}{c}0.01 \\
8\end{array}$ & $\begin{array}{c}2.91(1.15 \\
7.38)\end{array}$ & 0.024 \\
\hline 40-49 & $\begin{array}{c}6.11(2.78 \\
13.44)\end{array}$ & $\begin{array}{c}0.00 \\
0\end{array}$ & $\begin{array}{c}6.67(2.80 \\
15.91)\end{array}$ & 0.000 \\
\hline 50-59 & $\begin{array}{c}15.63(7.42 \\
32.92)\end{array}$ & $\begin{array}{c}0.00 \\
0\end{array}$ & $\begin{array}{c}17.49 \text { (7.43, } \\
41.15)\end{array}$ & 0.000 \\
\hline 60-69 & $\begin{array}{c}6.96(2.6 \\
18.63)\end{array}$ & $\begin{array}{c}0.00 \\
0\end{array}$ & $\begin{array}{c}6.7(2.18 \\
20.63)\end{array}$ & 0.001 \\
\hline \multicolumn{5}{|l|}{ Physical activity } \\
\hline Sufficient & 1.00 & & 1.00 & \\
\hline Insufficient & $\begin{array}{c}2.51(1.37 \\
4.60)\end{array}$ & $\begin{array}{c}0.00 \\
3\end{array}$ & $\begin{array}{c}2.78(1.41 \\
5.47)\end{array}$ & 0.003 \\
\hline \multicolumn{5}{|l|}{ BMI } \\
\hline Normal & 1.00 & & 1.00 & \\
\hline Overweight & $\begin{array}{c}3.31(1.87 \\
5.85)\end{array}$ & $\begin{array}{c}0.00 \\
0\end{array}$ & $\begin{array}{c}2.25(1.19 \\
4.26)\end{array}$ & 0.013 \\
\hline \multicolumn{5}{|l|}{ Waist Hip Ratio } \\
\hline Normal & 1.00 & & 1.00 & \\
\hline Centrally obese & $\begin{array}{c}3.00(1.80 \\
5.01)\end{array}$ & $\begin{array}{c}0.00 \\
0\end{array}$ & $\begin{array}{c}1.53(0.85 \\
2.74)\end{array}$ & 0.157 \\
\hline
\end{tabular}

The odds of prediabetes in individuals aged 50-59 was 2.21 times that in younger individuals aged 18 to 29 years The odds of overweight was 1.44 times while obese individuals was more than double at 2.55 times that in normal-weight individuals
Conclusions

- This is the first nationally representative study that has quantified the burden of diabetes in Kenya

- Prevalence of prediabetes is triple the prevalence of diabetes

Pre-diabetes and diabetes is more prevalent in women than men

- Predictors of prediabetes are age of 50-69, non heavy episodic drinking, central obesity, overweight and obesity

- Predictors of diabetes are age group of 50-59, insufficient physical activity, overweight and central obesity Limitations

use self-reported measures for behavioral risk factors possible biases from incomplete data due to nonrespondents

Recommendations Introducing innovative public health interventions through policy development

Promoting adoption of healthy lifestyles.

References

IDF. (2013). International Diabetes Federation. IDF diabetes atlas. 6th ed. Retrieved from ttps://www.idf.org/sites/default /files/EN_6E_Atlas_Full_0.pdf Marquez, P. V, \& Farrington, J. L. (2013). The Challenge of NonCommunicable Diseases and Road Traffic Injuries in Sub-Saharan Africa AN OVERVIEW, 108. Retrieved from

http://ncdalliance.org/sites/defa ult/files/The Challenge of NonCommunicable Diseases and Road Traffic Injuries in Sub-Saharan Africa - AN OVERVIEW.pdf

WHO. (2008a). Chapter 1 Burden : mortality , morbidity and risk factors.

Acknowledgements

African Population and Health Research Center Ministry of Health WHO

Kenya National Bureau of statistics

Kenya Medical Research Center

African Institute for Health and Development 